\title{
On the Role of Screening for Intracranial Aneurysms in Autosomal Dominant Polycystic Kidney Disease
}

$I^{n+m}$

tracranial aneurysm (ICAN) formation is a well-known complication in patients with autosomal dominant polycystic kidney disease (ADPKD). In these patients, aneurysms are thought to form as a consequence of abnormal polycystin protein expression in vascular myocytes, which impairs the structural integrity of vessel walls. ${ }^{1-4}$ ICAN rupture with SAH is the most serious morbidity associated with ADPKD.

ICANs, both in ADPKD and the general population, are acquired abnormalities that are thought to form during a relatively short time. A defect in the elastic lamina of a vessel permits outward bulging, the extent of which is determined by the opposing forces of arterial blood pressure and residual elasticity of the vessel. A new aneurysm will either rupture or stabilize due to collagenous scarring. Aneurysms smaller than approximately $7 \mathrm{~mm}$ are less likely to rupture initially and may be less likely to expand with time. Because ICAN formation in ADPKD is associated with a defective vessel wall protein, the time course and dynamics of aneurysm stabilization and growth could potentially be altered. Most interesting, ICAN formation in ADPKD is not dependent on the presence of arterial hypertension, ${ }^{5,6}$ though ICAN rupture is.

The value of widespread radiographic screening for ICANs in asymptomatic patients with ADPKD is controversial, mainly because of the potential risk involved in pre-emptively treating incidentally detected aneurysms. Knowing which patients are more likely to have aneurysmal SAH could help stratify high-risk patients toward endovascular or surgical aneurysm repair and lowrisk patients toward counseling and periodic imaging surveillance. ${ }^{7}$ One limitation of screening is that aneurysms that rupture around the time of their formation could be missed by infrequent imaging.

Regarding screening of asymptomatic patients, 3D time-offlight MR angiography is the technique of choice because it avoids the risk assumed with contrast-based angiography, including iodinated contrast-induced nephropathy ${ }^{8}$ and gadolinium contrast-induced nephrogenic systemic fibrosis, ${ }^{9}$ as well as complications related to catheter-based angiography. ${ }^{10}$ For acutely symptomatic patients, urgent CT or catheter-based angiography should be performed.
Although the prevalence of ICANs in ADPKD increases with age, small studies have not demonstrated an increased rate of aneurysm growth and rupture compared with age-matched nonADPKD populations. ${ }^{11,12}$ The risk of aneurysm rupture in ADPKD is linked to a family history of ICAN and prior SAH. ${ }^{5,6,13}$ In a retrospective study of 369 patients with ADPKD, the mean age of aneurysmal SAH in ADPKD was found to be slightly less than that in the general population (53 compared with 57 years of age). ${ }^{5}$ Additional risk factors for aneurysm rupture are extrapolated from the International Study of Unruptured Intracranial Aneurysms (ISUIA) ${ }^{14}$ and include aneurysm features such as size, shape, and location, as well as the presence of neurologic symptoms or signs. Now, patients with ADPKD with a family history of ICAN or prior SAH are routinely screened.

For patients with ADPKD without a family history of ICAN or prior SAH, the benefit of screening is less clear, and there are small clinical studies and meta-analyses advocating for ${ }^{6,15}$ and against $^{11,12}$ routine screening. In the largest of these studies, which examined a cohort of 355 patients in China, ${ }^{6}$ the prevalence of ICAN increased with age, from $6.0 \%$ in the 30 - to 39 -year age group to $23.3 \%$ in the 60 - to 69 -year age group. On the basis of these findings, the authors favored screening all patients with ADPKD 30 years of age or older.

Niemczyk et $\mathrm{al}^{16}$ examined the prevalence of ICAN in ADPKD in a population of 83 adult patients in Poland. The data presented in this patient group were roughly concordant with those in other studies in terms of overall ICAN prevalence in ADPKD and increased ICAN prevalence with age. No risk factors were identified to predict ICAN rupture in younger patients. As in the study by $\mathrm{Xu}$ et $\mathrm{al},{ }^{6}$ the authors recommended screening all patients older than 45 years of age.

A recommendation to preferentially screen older patients with ADPKD should be balanced by safety analyses of potential endovascular or surgical interventions. Elevated morbidity and mortality rates in patients older than 50 years of age undergoing craniotomy and surgical clipping and older than 70 years of age undergoing endovascular coiling have been reported. ${ }^{17}$ Older patients with ADPKD commonly develop renal failure as well as arterial hypertension and may have even greater postoperative 
complications compared with younger patients with ADPKD or age-matched patients without ADPKD.

The economics of screening has been studied as well. A decision analysis of aneurysm screening versus nonscreening in $\mathrm{ADPKD}^{15}$ showed the greatest benefit in younger patients, in whom identification and treatment of an asymptomatic aneurysm increased life expectancy. As stated above, however, ICAN rupture in young patients is considerably rarer than in older patients. A risk analysis of treatment versus nontreatment based on ISUIA data ${ }^{18}$ did not favor clipping of unruptured aneurysms of $<10 \mathrm{~mm}$, though analyses of potential life-years saved in subgroups including ADPKD could not be calculated because the natural history and treatment risks in these subgroups are unknown.

The principles of a screening test, as originally described by the World Health Organization, include an understanding of the natural history of the disease in question as well as consensus on whom to treat. ${ }^{19}$ Whether the natural history of aneurysm formation, growth, and rupture risk in ADPKD parallels that of the general population remains unsettled. More data are needed from the ADPKD population to generate evidence-based guidelines for radiographic screening.

\section{REFERENCES}

1. Griffin MD, Torres VE, Grande JP, et al. Vascular expression of polycystin. J Am Soc Nephrol 1997;8:616-26

2. Torres VE, Cai Y, Chen X, et al. Vascular expression of polycystin-2. J Am Soc Nephrol 2001;12:1-9

3. Zhang B, Fugleholm K, Day LB, et al. Molecular pathogenesis of subarachnoid haemorrhage. Int J Biochem Cell Biol 2003;35:1341-60

4. Brisman JL, Song JK, Newell DW. Cerebral aneurysms. N Engl J Med 2006;355:928-39

5. Gieteling EW, Rinkel GJ. Characteristics of intracranial aneurysms and subarachnoid hemorrhage in patients with polycystic kidney disease. J Neurol 2003;250:418-23

6. Xu HW, Yu SQ, Mei CL, et al. Screening for intracranial aneurysm in 355 patients with autosomal-dominant polycystic kidney disease. Stroke 2011;42:204-06

7. Rinkel GJ. Intracranial aneurysm screening: indications and advice for practice. Lancet Neurol 2005;4:122-28

8. Weisbord SD, Palevsky PM. Strategies for the prevention of con- trast-induced acute kidney injury. Curr Opin Nephrol Hypertens 2010;19:539-49

9. Mayr M, Burkhalter F, Bongartz G. Nephrogenic systemic fibrosis: clinical spectrum of disease. J Mag Reson Imaging 2009;30:1289-97

10. Willinsky RA, Taylor SM, TerBrugge K, et al. Neurologic complications of cerebral angiography: prospective analysis of 2,899 procedures and review of the literature. Radiology 2003;227:522-28

11. Gibbs GF, Huston J 3rd, Qian Q, et al. Follow-up of intracranial aneurysms in autosomal-dominant polycystic kidney disease. Kidney Int 2004;65:1621-27

12. Irazabal MV, Huston J 3rd, Kubly V, et al. Extended follow-up of unruptured intracranial aneurysms detected by presymptomatic screening in patients with autosomal dominant polycystic kidney disease. Clin J Am Soc Nephrol 2011;6:1274-85

13. Vlak MH, Algra A, Brandenburg R, et al. Prevalence of unruptured intracranial aneurysms, with emphasis on sex, age, comorbidity, country, and time period: a systematic review and meta-analysis. Lancet Neurol 2011;10:626-36

14. Wiebers DO, Whisnant JP, Huston J 3rd, et al, for the International Study of Unruptured Intracranial Aneurysms Investigators. Unruptured intracranial aneurysms: natural history, clinical outcome, and risks of surgical and endovascular treatment. Lancet 2003;362:103-10

15. Butler WE, Barker FG 2nd, Crowell RM. Patients with polycystic kidney disease would benefit from routine magnetic resonance angiographic screening for intracerebral aneurysms: a decision analysis. Neurosurgery 1996;38:506-16, discussion 515-16

16. Niemczyk M, GradzikM, Niemczyk S, et al. Intracranial aneurysms in autosomal dominant polycystic kidney disease. AJNR Am J Neuroradiol 2013;34:1556-59.

17. Wiebers DO, Piepgras DG, Meyer FB, et al. Pathogenesis, natural history, and treatment of unruptured intracranial aneurysms. Mayo Clin Proc 2004;79:1572-83

18. Mitchell P, Jakubowski J. Risk analysis of treatment of unruptured aneurysms. J Neurol Neurosurg Psychiatry 2000;68:577-80

19. Wilson JM, Jungner G. World Health Organization. Principles and Practice of Screening for Disease. Public Health Papers No. 34. Geneva, Switzerland; World Health Organization; 1968:1-163. http:// whqlibdoc.who.int/php/WHO_PHP_34.pdf. Accessed January 12, 2013

J.P. Klein

Departments of Neurology and Radiology Brigham and Women's Hospital and Harvard Medical School Boston, Massachusetts

http://dx.doi.org/10.3174/ajnr.A3466 